\title{
Synthesis and Determination of the Absolute Configuration of Chiral Tetracosanaphthalenes.
}

\author{
Kazunori Tsubaki,* Kazuto Takaishi, Daisuke Sue, Takeo Kawabata \\ Institute for Chemical Research, Kyoto University, Gokasho, Uji, Kyoto, 611-0011, Japan.
}

Email tsubaki@fos.kuicr.kyoto-u.ac.jp

Pages 2-8: Synthesis of the Oligonaphthalene Derivatives.

Page 9: Application of the exciton chirality method to 16mers.

Pages 10-23: ${ }^{1} \mathrm{H}-\mathrm{NMR}$ charts for compounds

Page 24: $\quad$ HPLC chart of the mixture of mono-ol 3 and diol 4. 


\section{Experimental Section}

General: Unless otherwise specified, all ${ }^{1} \mathrm{H}-\mathrm{NMR}$ spectra were taken at 200, 300 or $400 \mathrm{MHz}$ in $\mathrm{CDCl}_{3}$ with chemical shifts being reported at $\delta \mathrm{ppm}$ from tetramethylsilane as an internal standard. Flash column chromatography was carried out with silica gel 60 spherical (150-325 mesh) and silica gel $60 \mathrm{~F}_{254}$ plates (Merck) for preparative TLC.

Synthesis of $(\boldsymbol{R}, \boldsymbol{R}, \boldsymbol{R}, \boldsymbol{R}, \boldsymbol{R}, \boldsymbol{R}, \boldsymbol{R})-2:$ To a suspension of $(R, R, R, R, R, R, R, R)-1(448 \mathrm{mg}, 0.19 \mathrm{mmol})$ and potassium carbonate $(79 \mathrm{mg}, 0.57 \mathrm{mmol}, 3.0$ eq. $)$ in DMF $(15 \mathrm{ml})$, iodomethane $(59 \mu \mathrm{l}, 0.95 \mathrm{mmol}, 5.0$ eq.) was added. The reaction mixture was stirred for 11 hours at room temperature. The reaction mixture was poured into the mixed solvent of ethyl acetate and water. The organic layer was separated, washed successively with $0.1 \mathrm{M}$ hydrochloric acid solution, water (twice) and brine. After dried over sodium sulfate, the solvent was evaporated in vacuo to afford pale yellow powder (465 mg, 100\%). Crude $(R, R, R, R, R, R, R)-\mathbf{2}$ was directly used for the next step without further purification. A small part of the sample was subjected to further purification by PTLC to give an analytical sample. yellow powder; $\mathrm{mp}=131{ }^{\circ} \mathrm{C} ;[\alpha]_{\mathrm{D}}{ }^{20}=+120.2\left(\mathrm{c}=0.50, \mathrm{CHCl}_{3}\right)$; IR $(\mathrm{KBr}) 2930,1642,1550,1446 \mathrm{~cm}^{-1} ;{ }^{1} \mathrm{H}^{-}$ NMR (200 MHz, $\left.\mathrm{CDCl}_{3}\right) \delta$ 0.5-1.2 (m, 48H), 2.6-3.4 (m, 32H), $3.84(\mathrm{~s}, 6 \mathrm{H}), 3.89(\mathrm{~s}, 6 \mathrm{H}), 3.91(\mathrm{~s}, 6 \mathrm{H})$, 4.4-5.1 (m, 16H), $5.38(\mathrm{~s}, 4 \mathrm{H}), 7.1-7.7(\mathrm{~m}, 42 \mathrm{H}), 7.84(\mathrm{~d}, J=8.2 \mathrm{~Hz}, 2 \mathrm{H})$; MS $\left(\mathrm{FAB}^{+}\right) \mathrm{m} / z 2436\left(\mathrm{M}^{+}\right)$, Anal. Calcd for $\mathrm{C}_{148} \mathrm{H}_{162} \mathrm{~N}_{8} \mathrm{O}_{24} \cdot 0.8 \mathrm{CH}_{3} \mathrm{Cl}: \mathrm{C}, 70.57 ; \mathrm{H}, 6.48 ; \mathrm{N}, 4.42$. Found: C, 70.34; H, 6.88; N, 4.17.

Synthesis of $(\boldsymbol{R}, \boldsymbol{R}, \boldsymbol{R}, \boldsymbol{R}, \boldsymbol{R}, \boldsymbol{R}, \boldsymbol{R})-3$ : To a suspension of $(R, R, R, R, R, R, R)-2(550 \mathrm{mg}, 0.23 \mathrm{mmol})$ and $10 \%$ palladium on carbon $(55 \mathrm{mg})$ in $\mathrm{MeOH}(20 \mathrm{ml})$, was stirred under hydrogen atmosphere for 16 hours at room temperature with monitoring the reaction by TLC (hexane/ethyl acetate/methanol $=3 / 5 / 1$ ). After palladium on carbon was filtered off, the filtrate was evaporated to give a residue. The residue was purified by PTLC $\left(\mathrm{SiO}_{2}\right.$, hexane/ethyl acetate/methanol = 4/5/1) to afford $(R, R, R, R, R, R, R)-3$ (169 $\mathrm{mg}, 33 \%)$ and recovered $(R, R, R, R, R, R, R)-2(72 \mathrm{mg}, 13 \%)$ and over-reacted $(R, R, R, R, R, R, R)-4(97 \mathrm{mg}$, 19\%). A small part of the sample was subjected to further purification by PTLC to give an analytical

sample. yellow powder; $\mathrm{mp}=111^{\circ} \mathrm{C} ;[\alpha]_{\mathrm{D}}{ }^{20}=+146.8\left(\mathrm{c}=0.50, \mathrm{CHCl}_{3}\right)$; IR $(\mathrm{KBr}) 2972,1642,1549$, $1446 \mathrm{~cm}^{-1} ;{ }^{1} \mathrm{H}-\mathrm{NMR}\left(200 \mathrm{MHz}, \mathrm{CDCl}_{3}\right) \delta$ 0.5-1.2 (m, 48H), 2.6-3.4 (m, 32H), 3.8-3.95 (m, 18H), 4.3$5.1(\mathrm{~m}, 16 \mathrm{H}), 5.34(\mathrm{~s}, 2 \mathrm{H}), 7.1-7.7(\mathrm{~m}, 37 \mathrm{H}), 7.8-7.9(\mathrm{~m}, 2 \mathrm{H}) 10.87(\mathrm{~s}, 1 \mathrm{H}) ; \mathrm{MS}\left(\mathrm{FAB}^{+}\right) \mathrm{m} / z 2368$ $(\mathrm{M}+\mathrm{Na})^{+}$.

Synthesis of deastereomeric mixture 16mer 5 and deastereomeric mixture 24mer 8: To a mixture of $\mathrm{CuCl}_{2}(43 \mathrm{mg}, 0.32 \mathrm{mmol})$ in methanol $(1.0 \mathrm{ml}), i$-propylamine $(34 \mu 1,0.40 \mathrm{mmol})$ was added under 
argon atmosphere at room temperature. After $1 \mathrm{~h}$, a solution of $(R, R, R, R, R, R, R)-3(70.7 \mathrm{mg}, 30 \mu \mathrm{mol})$ and $(R, R, R, R, R, R, R)-\mathbf{4}(4.3 \mathrm{mg}, 2 \mu \mathrm{mol})$ in dichloromethane $(1 \mathrm{ml})$ was added and the reaction mixture was stirred for $11 \mathrm{~h}$ at room temperature. The reaction mixture was poured into the mixed solvent of 0.1 M hydrochloric acid solution and chloroform. The organic layer was washed successively with $0.1 \mathrm{M}$ hydrochloric acid solution, water and brine, and dried over sodium sulfate and evaporated to give a residue. The residue was purified by recycling preparative HPLC connected with JAIGEL-1H $(20 \mathrm{x}$ $600 \mathrm{~mm})$ and JAIGEL-2H $(20 \times 600 \mathrm{~mm})$ under the conditions of $3.5 \mathrm{ml} / \mathrm{min}$ of flow rate with $\mathrm{CHCl}_{3}$ detected by UV (254 nm) to afford a diastereomeric mixture of 16mer 5 (35 mg, 37\% based of 3), diastereomeric mixture of $24 \mathrm{mer} \mathbf{8}(8 \mathrm{mg}, 60 \%$ based of 4$)$ and recovered $\mathbf{3}$ (29 $\mathrm{mg}, 24 \%)$.

Synthesis and separation of diastereomer 6: To a suspension of diatereomeric mixture 5 (63 mg, $0.013 \mathrm{mmol})$ and potassium carbonate $(5 \mathrm{mg}, 0.039 \mathrm{mmol}, 3.0$ eq. $)$ in DMF $(2 \mathrm{ml})$, iodomethane $(4 \mu \mathrm{l}$, $0.065 \mathrm{mmol}, 5.0$ eq.) was added. The rection mixture was stirred for 36 hours at room temperature. The reaction mixture was poured into the mixed solvent of chloroform and water. The organic layer was separated, washed successively with $0.1 \mathrm{M}$ hydrochloric acid solution, water (twice) and brine. After dried over sodium sulfate, the solvent was evaporated in vacuo to afford yellow powder 6 (54 mg, 0.011 mmol, 88\%) as a diastereomeric mixture. Diastereomeric mixture 6 (30 mg, $6.3 \mu \mathrm{mol})$ was separated by recycling preparative HPLC connected with COSMOSIL 5SL-II (20 x $250 \mathrm{~mm}$, nacalai tesque) under the condition of $5 \mathrm{ml} / \mathrm{min}$ of flow rate with eluent (ethyl acetate/ ethanol =8/2) detected by UV $(254 \mathrm{~nm})$ to afford $(R, R, R, R, R, R, R, \underline{\boldsymbol{R}}, R, R, R, R, R, R, R)-6\left(9 \mathrm{mg}, 1.9 \mu \mathrm{mol}, 30 \%, t_{R}=16.2 \mathrm{~min}\right)$ and $(R, R, R, R, R, R, R, \underline{S}, R, R, R, R, R, R, R)-6\left(10 \mathrm{mg}, 2.1 \mu \mathrm{mol}, 33 \%, t_{R}=15.1 \mathrm{~min}\right)$.

\section{$(R, R, R, R, R, R, R, R, R, R, R, R, R, R, R)-6$}

yellow powder; $\mathrm{mp}>300{ }^{\circ} \mathrm{C} ;[\alpha]_{\mathrm{D}}{ }^{20}=+114\left(\mathrm{c}=0.53, \mathrm{CHCl}_{3}\right)$; IR $(\mathrm{KBr}) 2971,2933,1655,1457 \mathrm{~cm}^{-1}$;

${ }^{1} \mathrm{H}-\mathrm{NMR}\left(400 \mathrm{MHz}, \mathrm{CDCl}_{3}\right) \delta 0.61(\mathrm{t}, J=7.2 \mathrm{~Hz}, 6 \mathrm{H}), 0.7-1.2(\mathrm{~m}, 90 \mathrm{H}), 2.5-3.4(\mathrm{~m}, 64 \mathrm{H}), 3.85(\mathrm{~s}, 6 \mathrm{H})$, 3.85-3.95 (m, 30H), $3.96(\mathrm{~s}, 6 \mathrm{H}), 4.32(\mathrm{~d}, J=12.0 \mathrm{~Hz}, 2 \mathrm{H}), 4.50(\mathrm{~d}, J=12.8 \mathrm{~Hz}, 2 \mathrm{H}), 4.5-5.1(\mathrm{~m}, 28 \mathrm{H})$, 5.38 (s, 4H), 7.2-7.7 (m, 74H), 7.83 (d, $J=8.4 \mathrm{~Hz}, 2 \mathrm{H})$; MALDI-TOF-MS (dithranol) 4720.74. 


\section{$(R, R, R, R, R, R, R, S, R, R, R, R, R, R, R)-6$}

yellow powder; $\mathrm{mp}>300{ }^{\circ} \mathrm{C} ;[\alpha]_{\mathrm{D}}^{20}=+108\left(\mathrm{c}=0.55, \mathrm{CHCl}_{3}\right)$; $\mathrm{IR}(\mathrm{KBr}) 2932,2370,1647,1458 \mathrm{~cm}^{-1}$;

${ }^{1} \mathrm{H}-\mathrm{NMR}\left(400 \mathrm{MHz}, \mathrm{CDCl}_{3}\right) \delta 0.61(\mathrm{t}, J=7.0 \mathrm{~Hz}, 6 \mathrm{H}), 0.7-1.2(\mathrm{~m}, 90 \mathrm{H}), 2.5-3.4(\mathrm{~m}, 64 \mathrm{H}), 3.85(\mathrm{~s}, 6 \mathrm{H})$, 3.9-4.0 (m, 36H), 4.32 (d, $J=12.0 \mathrm{~Hz}, 2 \mathrm{H}), 4.5-5.1(\mathrm{~m}, 30 \mathrm{H}), 5.38$ (s, 4H), 7.2-7.7 (m, 74H), 7.83 (d, $J$ $=8.4 \mathrm{~Hz}, 2 \mathrm{H}$ ); MALDI-TOF-MS (dithranol) 4720.30 .

\section{General Procedure for the Synthesis of Bisporphyrin Compounds} $(R, R, R, R, R, R, R, R, R, R, R, R, R, R, R)-7$ and $(R, R, R, R, R, R, R, S, R, R, R, R, R, R, R)-7:$ The synthesis of $(R, R, R, R, R, R, R, R, R, R, R, R, R, R, R)-7 \quad$ is typical. $\quad$ To a suspension of dibenzyl ether of $(R, R, R, R, R, R, R, R, R, R, R, R, R, R, R)-6(10 \mathrm{mg}, 2.1 \mu \mathrm{mol})$ and $10 \% \mathrm{Pd} / \mathrm{C}(2 \mathrm{mg})$ in methanol (4 ml) was added a drop of $4 \mathrm{~N} \mathrm{HCl} /$ ethyl acetate and stirred at room temperature for 45 hours under $\mathrm{H}_{2}$ balloon. The $\mathrm{Pd} / \mathrm{C}$ was filtered off and evaporated in vacuo to give a residue $(9 \mathrm{mg})$. To a solution of this residue in $\mathrm{CH}_{2} \mathrm{Cl}_{2}(2 \mathrm{ml})$, TPP- $\mathrm{CO}_{2} \mathrm{H}(5 \mathrm{mg}, 7.0 \mu \mathrm{mol})$, WSC.HCl $(2 \mathrm{mg}, 0.011 \mathrm{mmol})$ and DMAP (4 $\mathrm{mg}, 0.035 \mathrm{mmol}$ ) were added under argon atmosphere and stirred at room temperature for 19 hours. The reaction mixture was quenched with water, extracted with $\mathrm{CHCl}_{3}$, washed successively with water and brine, dried over $\mathrm{Na}_{2} \mathrm{SO}_{4}$ and evaporated to give a residue which was purified by GPC to afford $(R, R, R, R, R, R, R, R, R, R, R, R, R, R, R)-7$ as a purple solid (10 mg, $1.7 \mu \mathrm{mol}, 84 \%)$.

$\mathrm{mp}>300{ }^{\circ} \mathrm{C} ;[\alpha]_{\mathrm{D}}{ }^{20}=-3902\left(\mathrm{c}=0.05, \mathrm{CHCl}_{3}\right) ; \mathrm{IR}(\mathrm{KBr}) 2930,1739,1644,1455 \mathrm{~cm}^{-1} ;{ }^{1} \mathrm{H}-\mathrm{NMR}(300$ $\left.\mathrm{MHz}, \mathrm{CDCl}_{3}\right) \delta-2.74(\mathrm{~s}, 4 \mathrm{H}), 0.6-1.3(\mathrm{~m}, 96 \mathrm{H}), 2.8-3.5(\mathrm{~m}, 64 \mathrm{H}), 3.8-4.1(\mathrm{~m}, 42 \mathrm{H}), 4.4-5.2(\mathrm{~m}, 32 \mathrm{H})$, 7.2-7.9 (m, 80H), 8.0-8.4 (m, 16H), $8.44(\mathrm{~d}, J=8.1 \mathrm{~Hz}, 4 \mathrm{H}), 8.78(\mathrm{~d}, J=8.1 \mathrm{~Hz}, 4 \mathrm{H}), 8.8-9.0(\mathrm{~m}, 16 \mathrm{H})$; MALDI-TOF-MS (dithranol) 5818.34.

\section{$(R, R, R, R, R, R, R, S, R, R, R, R, R, R, R)-7$}

$53 \%$ yield; purple solid, $\mathrm{mp}>300{ }^{\circ} \mathrm{C} ;[\alpha]_{\mathrm{D}}{ }^{20}=-3824\left(\mathrm{c}=0.05, \mathrm{CHCl}_{3}\right)$; IR $(\mathrm{KBr}) 2929,1737,1648$, $1457 \mathrm{~cm}^{-1} ;{ }^{1} \mathrm{H}-\mathrm{NMR}\left(300 \mathrm{MHz}, \mathrm{CDCl}_{3}\right) \delta-2.75(\mathrm{~s}, 4 \mathrm{H}), 0.6-1.3(\mathrm{~m}, 96 \mathrm{H}), 2.8-3.5(\mathrm{~m}, 64 \mathrm{H}), 3.8-4.1(\mathrm{~m}$, $42 \mathrm{H}), 4.4-5.2(\mathrm{~m}, 32 \mathrm{H}), 7.2-7.9(\mathrm{~m}, 80 \mathrm{H}), 8.0-8.4(\mathrm{~m}, 16 \mathrm{H}), 8.44(\mathrm{~d}, J=8.1 \mathrm{~Hz}, 4 \mathrm{H}), 8.73(\mathrm{~d}, J=8.1 \mathrm{~Hz}$, 4H), 8.8-9.0 (m, 16H); MALDI-TOF-MS (dithranol) 5819.20.

Separation of diastereomer 9; Diastereomeric mixture $8(15.0 \mathrm{mg}, 2.2 \mu \mathrm{mol})$ was purified by recycling preparative HPLC connected with COSMOSIL 5SL-II (20 x $250 \mathrm{~mm}$, nacalai tesque) under the condition of $5 \mathrm{ml} / \mathrm{min}$ of flow rate with eluent (ethyl acetate/ ethanol $=8 / 2)$ detected by UV $(254$ $\mathrm{nm})$ to afford $(R, R, R, R, R, R, R, R, R, R, R, R, R, R, R, R, R, R, R, R, R, R, R)-9\left(2.2 \mathrm{mg}, 0.32 \mu \mathrm{mol}, 15 \%, t_{R}=16.7\right.$ $\min ),(R, R, R, R, R, R, R, S, R, R, R, R, R, R, R, R, R, R, R, R, R, R, R)-9\left(1.8 \mathrm{mg}, 0.26 \mu \mathrm{mol}, 12 \%, t_{R}=16.3 \mathrm{~min}\right)$ and $(R, R, R, R, R, R, R, S, R, R, R, R, R, R, R, S, R, R, R, R, R, R, R)-9\left(6.2 \mathrm{mg}, 0.86 \mu \mathrm{mol}, 41 \%, t_{R}=15.5 \mathrm{~min}\right)$. 


\section{$(R, R, R, R, R, R, R, R, R, R, R, R, R, R, R, R, R, R, R, R, R, R, R)-9$}

yellow powder; $\mathrm{mp}>300^{\circ} \mathrm{C} ;[\alpha]_{\mathrm{D}}^{20}=+121\left(\mathrm{c}=0.11, \mathrm{CHCl}_{3}\right)$; IR $(\mathrm{KBr}) 2925,2854,1647,1455 \mathrm{~cm}^{-1}$;

${ }^{1} \mathrm{H}-\mathrm{NMR}\left(300 \mathrm{MHz}, \mathrm{CDCl}_{3}\right) \delta$ 0.6-1.2 (m, 144H), 2.5-3.4 (m, 96H), 3.8-4.1 (m, 54H), 4.3-5.1 (m, 48H), $5.38(\mathrm{~s}, 4 \mathrm{H}), 7.2-7.7(\mathrm{~m}, 106 \mathrm{H}), 7.83(\mathrm{~d}, J=8.4 \mathrm{~Hz}, 2 \mathrm{H}), 10.61(\mathrm{~s}, 2 \mathrm{H}), 10.74(\mathrm{~s}, 2 \mathrm{H}) ;$ MALDI-TOF-MS (dithranol) 6946.00.

\section{$(R, R, R, R, R, R, R, R, R, R, R, R, R, R, R, S, R, R, R, R, R, R, R)-9$}

white powder; $\mathrm{mp}>300{ }^{\circ} \mathrm{C} ;[\alpha]_{\mathrm{D}}{ }^{20}=+120\left(\mathrm{c}=0.080, \mathrm{CHCl}_{3}\right)$; IR $(\mathrm{KBr}) 2922,1648,1456 \mathrm{~cm}^{-1} ;{ }^{1} \mathrm{H}-$ NMR $\left(300 \mathrm{MHz}, \mathrm{CDCl}_{3}\right) \delta$ 0.6-1.2 (m, 144H), 2.5-3.4 (m, 96H), 3.8-4.1 (m, 54H), 4.3-5.1 (m, 48H), $5.38(\mathrm{~s}, 4 \mathrm{H}), 7.2-7.7(\mathrm{~m}, 106 \mathrm{H}), 7.83(\mathrm{~d}, J=8.4 \mathrm{~Hz}, 2 \mathrm{H}), 10.62(\mathrm{~s}, 2 \mathrm{H}), 10.75(\mathrm{~s}, 2 \mathrm{H})$; MALDI-TOF-MS (dithranol) 6945.77 .

\section{$(R, R, R, R, R, R, R, S, R, R, R, R, R, R, R, S, R, R, R, R, R, R, R)-9$}

white powder; $\mathrm{mp}>300{ }^{\circ} \mathrm{C} ;[\alpha]_{\mathrm{D}}{ }^{20}=+116\left(\mathrm{c}=0.30, \mathrm{CHCl}_{3}\right)$; IR $(\mathrm{KBr}) 2925,1648,1459 \mathrm{~cm}^{-1} ;{ }^{1} \mathrm{H}-$ NMR $\left(300 \mathrm{MHz}, \mathrm{CDCl}_{3}\right) \delta$ 0.6-1.2 (m, 144H), 2.5-3.4 (m, 96H), 3.8-4.1 (m, 54H), 4.3-5.1 (m, 48H), $5.38(\mathrm{~s}, 4 \mathrm{H}), 7.2-7.7(\mathrm{~m}, 106 \mathrm{H}), 7.84(\mathrm{~d}, J=8.1 \mathrm{~Hz}, 2 \mathrm{H}), 10.62(\mathrm{~s}, 2 \mathrm{H}), 10.75(\mathrm{~s}, 2 \mathrm{H})$; MALDI-TOF-MS (dithranol) 6943.05.

General Procedure for the Synthesis of three kinds of tetraporphyrin Compounds (10). The synthesis of $(R, R, R, R, R, R, R, R, R, R, R, R, R, R, R, R, R, R, R, R, R, R, R)-\mathbf{1 0}$ is typical. To a solution of $(R, R, R, R, R, R, R, R, R, R, R, R, R, R, R, R, R, R, R, R, R, R, R)-9(2.2 \mathrm{mg}, 0.32 \mu \mathrm{mol})$ in $\mathrm{CH}_{2} \mathrm{Cl}_{2}(1 \mathrm{ml}), \mathrm{TPP}_{-} \mathrm{CO}_{2} \mathrm{H}$ (4.2 mg, $6.4 \mu \mathrm{mol})$, WSC.HCl $(1.2 \mathrm{mg}, 6.4 \mu \mathrm{mol})$ and DMAP $(0.8 \mathrm{mg}, 6.4 \mu \mathrm{mol})$ were added under argon atmosphere and stirred at room temperature for 2 weeks. The reaction mixture was quenched with water, extracted with $\mathrm{CHCl}_{3}$, washed successively with water and brine, dried over $\mathrm{Na}_{2} \mathrm{SO}_{4}$ and evaporated to give a residue which was purified by GPC to afford $(R, R, R, R, R, R, R, R, R, R, R, R, R, R, R, R, R, R, R, R, R, R, R)-10$ as a purple solid (1.0 mg, $1.1 \mu \mathrm{mol}, 33 \%)$.; $\mathrm{mp}>$ $300{ }^{\circ} \mathrm{C} ;[\alpha]_{\mathrm{D}}{ }^{20}=-1571\left(\mathrm{c}=0.06, \mathrm{CHCl}_{3}\right) ; \mathrm{IR}(\mathrm{KBr}) 2971,2934,1736,1637,1439 \mathrm{~cm}^{-1} ;{ }^{1} \mathrm{H}-\mathrm{NMR}(400$ $\left.\mathrm{MHz}, \mathrm{CDCl}_{3}\right) \delta-2.8(\mathrm{~m}, 8 \mathrm{H}), 0.6-1.3(\mathrm{~m}, 144 \mathrm{H}), 2.6-3.6(\mathrm{~m}, 96 \mathrm{H}), 3.8-4.1(\mathrm{~m}, 54 \mathrm{H}), 4.4-5.2(\mathrm{~m}, 48 \mathrm{H})$, $5.37(\mathrm{~s}, 4 \mathrm{H}), 7.2-7.9(\mathrm{~m}, 136 \mathrm{H}), 8.0-8.4(\mathrm{~m}, 32 \mathrm{H}), 8.4-9.0(\mathrm{~m}, 48 \mathrm{H})$; MALDI-TOF-MS (dithranol) 9505.21 . 
$42 \%$ yield; purple solid, $\mathrm{mp}>300{ }^{\circ} \mathrm{C} ;[\alpha]_{\mathrm{D}}{ }^{20}=-3214\left(\mathrm{c}=0.05, \mathrm{CHCl}_{3}\right)$; IR $(\mathrm{KBr}) 2969,2931,1736$, $1639 \mathrm{~cm}^{-1} ;{ }^{1} \mathrm{H}-\mathrm{NMR}\left(400 \mathrm{MHz}, \mathrm{CDCl}_{3}\right) \delta-2.8$ (brs, 8H), 0.6-1.3 (m, 144H), 2.6-3.6 (m, 96H), 3.8-4.1 $(\mathrm{m}, 54 \mathrm{H}), 4.4-5.2(\mathrm{~m}, 48 \mathrm{H}), 5.36(\mathrm{~s}, 4 \mathrm{H}), 7.2-7.9(\mathrm{~m}, 136 \mathrm{H}), 8.0-8.4(\mathrm{~m}, 32 \mathrm{H}), 8.4-9.0(\mathrm{~m}, 48 \mathrm{H})$; MALDI-TOF-MS (dithranol, M+Na) 9524.15.

\section{$(R, R, R, R, R, R, R, S, R, R, R, R, R, R, R, S, R, R, R, R, R, R, R)-10$}

$85 \%$ yield; purple solid, $\mathrm{mp}>300{ }^{\circ} \mathrm{C} ;[\alpha]_{\mathrm{D}}{ }^{20}=-1491\left(\mathrm{c}=0.12, \mathrm{CHCl}_{3}\right)$; IR $(\mathrm{KBr}) 2967,2931,1739$, $1645,1442 \mathrm{~cm}^{-1} ;{ }^{1} \mathrm{H}-\mathrm{NMR}\left(400 \mathrm{MHz}, \mathrm{CDCl}_{3}\right) \delta-2.70(\mathrm{~s}, 8 \mathrm{H}), 0.6-1.3(\mathrm{~m}, 144 \mathrm{H}), 2.6-3.6(\mathrm{~m}, 96 \mathrm{H}), 3.8-$ $4.1(\mathrm{~m}, 54 \mathrm{H}), 4.4-5.2(\mathrm{~m}, 48 \mathrm{H}), 5.38(\mathrm{~s}, 4 \mathrm{H}), 7.2-7.9(\mathrm{~m}, 136 \mathrm{H}), 8.0-8.4(\mathrm{~m}, 32 \mathrm{H}), 8.4-9.0(\mathrm{~m}, 48 \mathrm{H})$; MALDI-TOF-MS (dithranol) 9500.42. 


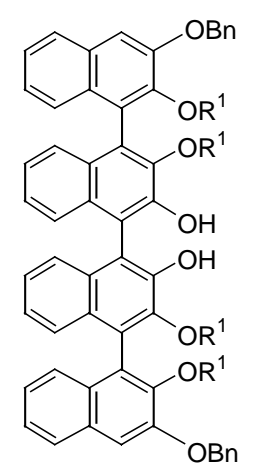

11

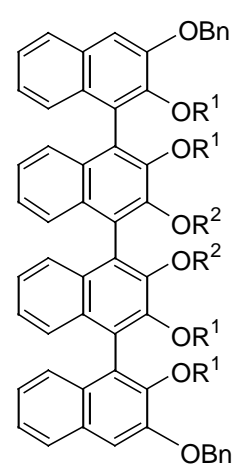

12

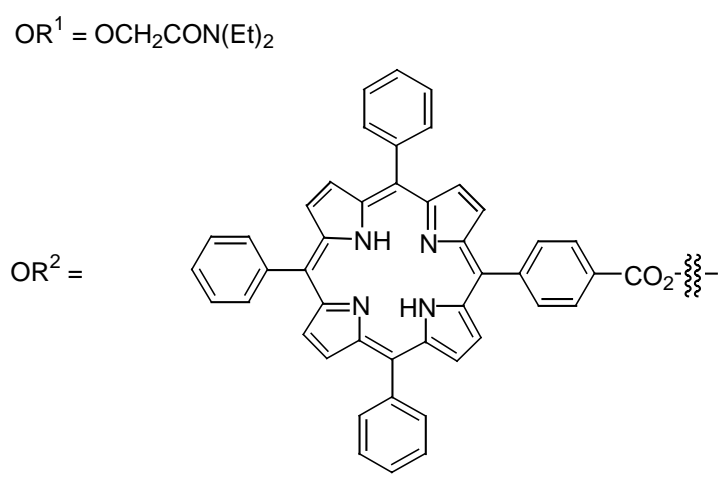

In order to certify the authenticity of configuration of tetracosanaphthalens by exciton chirality method, compounds $(S, S, S)$ and $(S, R, S)-\mathbf{1 2}$ which were already determined axial chiralities, were synthesized and applied this method. Compounds 12 were synthesized in the same way of synthesis of 7 except used compound $\mathbf{1 1}$ as starting materials.

(S,S,S)-11: known; Tsubaki, K. et. al. J. Org. Chem., 2006, 71, 6579-6587.

(S,R,S)-11: known; Tsubaki, K. et. al. J. Org. Chem., 2006, 71, 6579-6587.

$(S, S, S)-12 ; 44 \%$ yield; purple solid, $\mathrm{mp}=195{ }^{\circ} \mathrm{C} ;[\alpha]_{\mathrm{D}}{ }^{20}=-4911\left(\mathrm{c}=0.036, \mathrm{CHCl}_{3}\right)$; IR $(\mathrm{KBr}) 3316$, 1743, 1645, $1441 \mathrm{~cm}^{-1} ;{ }^{1} \mathrm{H}-\mathrm{NMR}\left(400 \mathrm{MHz} \mathrm{CDCl}_{3}\right) \delta-2.82(\mathrm{~s}, 4 \mathrm{H}), 0.55$ (brs, 6H), $0.83(\mathrm{t}, J=9.2,6 \mathrm{H})$, $0.87(\mathrm{t}, J=6.8,6 \mathrm{H}), 1.07(\mathrm{t}, J=7.2,6 \mathrm{H}), 2.9-3.4(\mathrm{~m}, 16 \mathrm{H}), 4.27(\mathrm{~d}, J=12.4,2 \mathrm{H}), 4.48$ (brs, $2 \mathrm{H}), 4.76$ $(\mathrm{d}, J=12.8,2 \mathrm{H}), 5.05(\mathrm{~d}, J=12.8,2 \mathrm{H}), 5.37(\mathrm{~s}, 4 \mathrm{H}), 7.2-7.8(\mathrm{~m}, 42 \mathrm{H}), 7.93$ (brs, 2H), 8.1-8.3 (m, 14H), $8.68(\mathrm{~d}, J=4.8,4 \mathrm{H}), 8.71(\mathrm{~d}, J=4.8,4 \mathrm{H}), 8.8-9.0(\mathrm{~m}, 16 \mathrm{H}) ; \mathrm{MS}\left(\mathrm{FAB}^{+}\right) 2570\left(\mathrm{M}+\mathrm{Na}^{+}\right)$.

$(\boldsymbol{S}, \boldsymbol{R}, \boldsymbol{S})-\mathbf{1 2} ; 47 \%$ yield; purple solid, $\mathrm{mp}=197{ }^{\circ} \mathrm{C} ;[\alpha]_{\mathrm{D}}{ }^{20}=-3276\left(\mathrm{c}=0.052, \mathrm{CHCl}_{3}\right) ; \mathrm{IR}(\mathrm{KBr}) 3316$, 1742, 1648, $1599 \mathrm{~cm}^{-1}$; ${ }^{1} \mathrm{H}-\mathrm{NMR}\left(400 \mathrm{MHz}, \mathrm{CDCl}_{3}\right) \delta-2.84(\mathrm{~s}, 4 \mathrm{H}), 0.5-1.0(\mathrm{~m}, 24 \mathrm{H}), 2.5-3.2(\mathrm{~m}, 16 \mathrm{H})$, $4.32(\mathrm{~d}, J=11.9,2 \mathrm{H}), 4.44$ (brs, 2H), 4.64 (d, $J=11.9,2 \mathrm{H}), 4.88$ (brs, 2H), 5.35 (s, 4H), 7.2-7.8 (m, $42 \mathrm{H}), 7.83(\mathrm{~d}, J=7.8,2 \mathrm{H}), 8.1-8.3(\mathrm{~m}, 14 \mathrm{H}), 8.33(\mathrm{~d}, J=7.3,4 \mathrm{H}), 8.56(\mathrm{~d}, J=6.4,4 \mathrm{H}), 8.8-9.0(\mathrm{~m}$, $16 \mathrm{H}) ; \mathrm{MS}\left(\mathrm{FAB}^{+}\right) 2570\left(\mathrm{M}+\mathrm{Na}^{+}\right)$. 


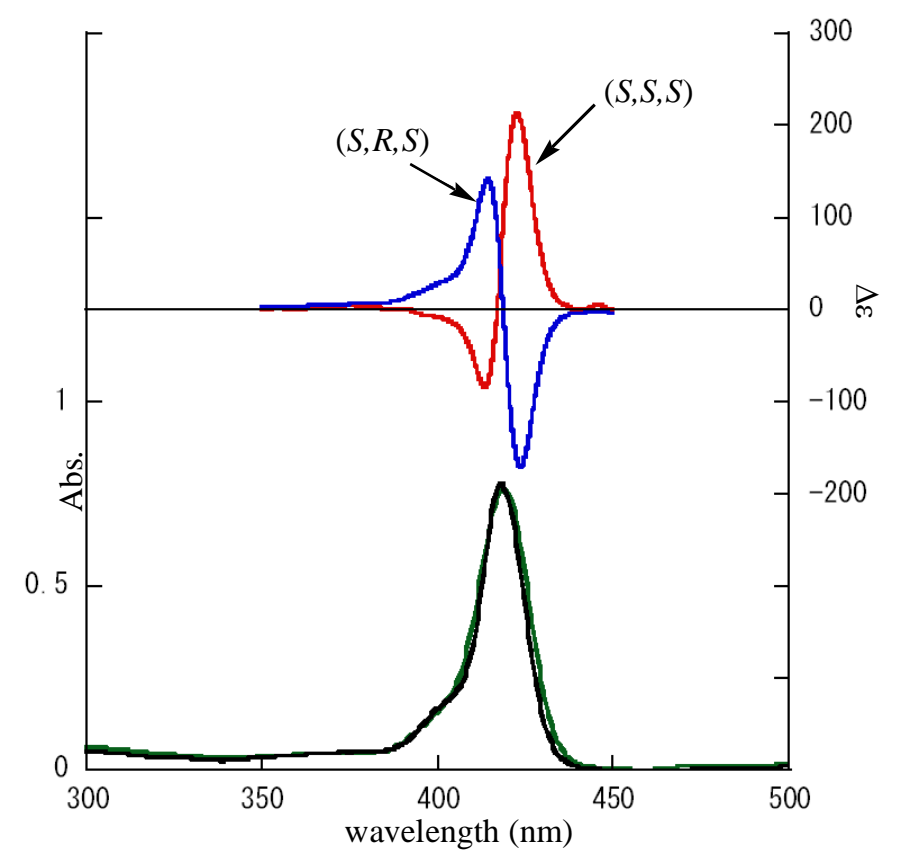

Figure S-1. UV-vis and CD spectra of 12: conditions: $\mathrm{CHCl}_{3}, 1.0 \times 10^{-5} \mathrm{M}, 25^{\circ} \mathrm{C}$, light path length $=1 \mathrm{~mm}$. 
Application of the exciton chirality method to 16 mers.

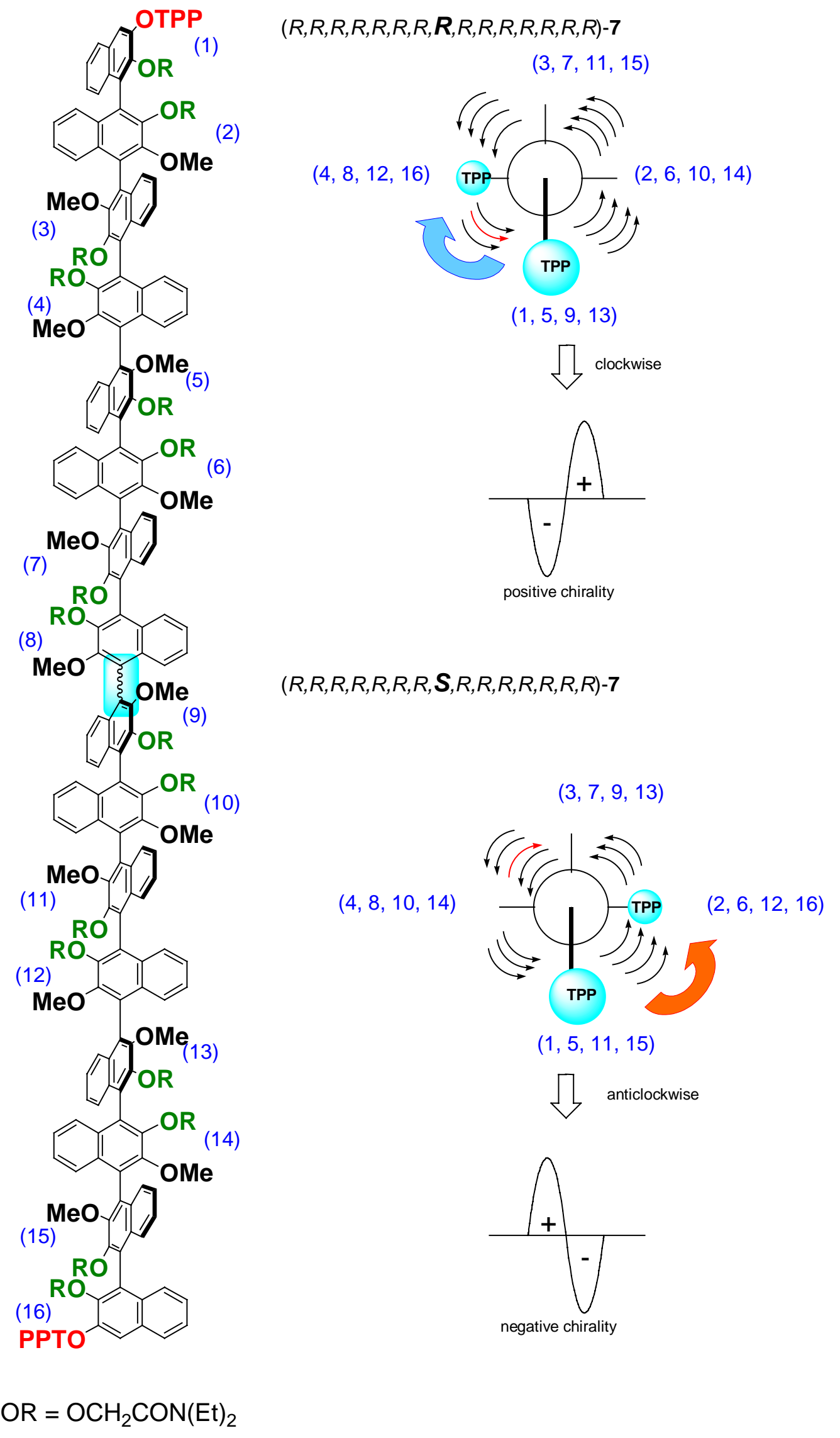




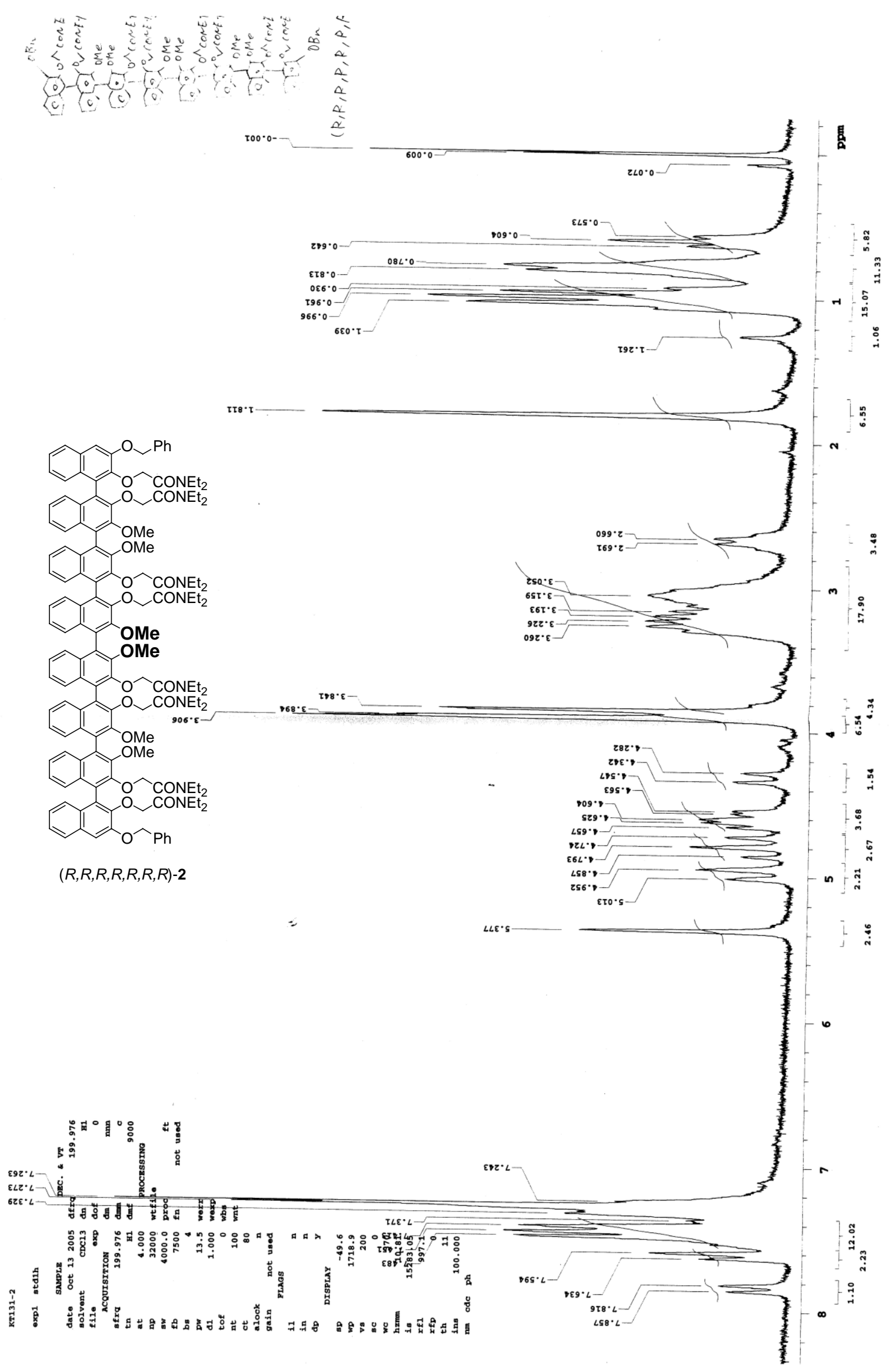




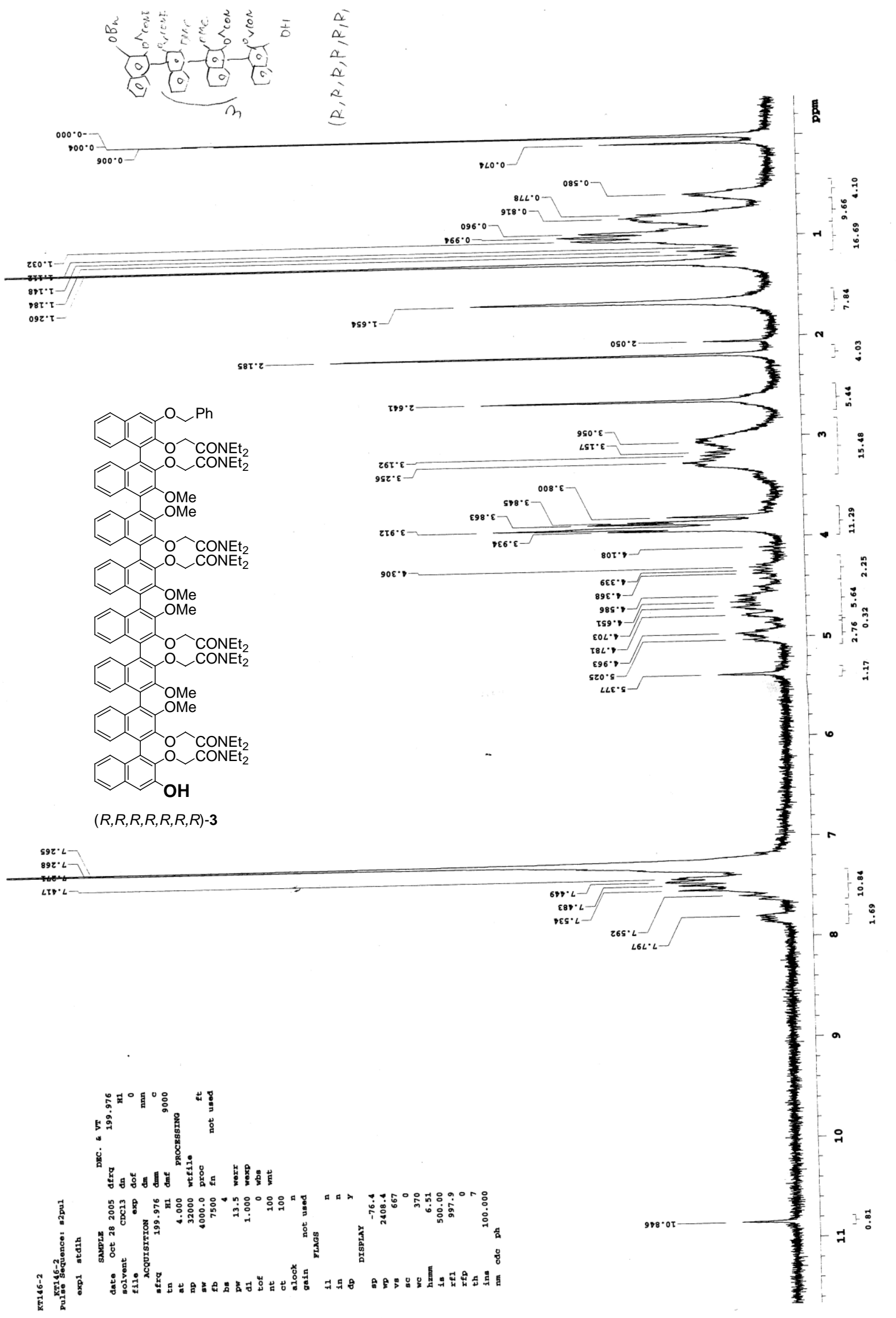




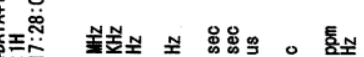

\$8

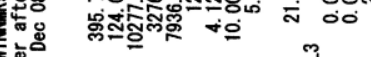

\pm 票

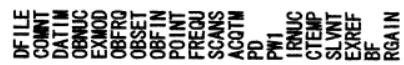

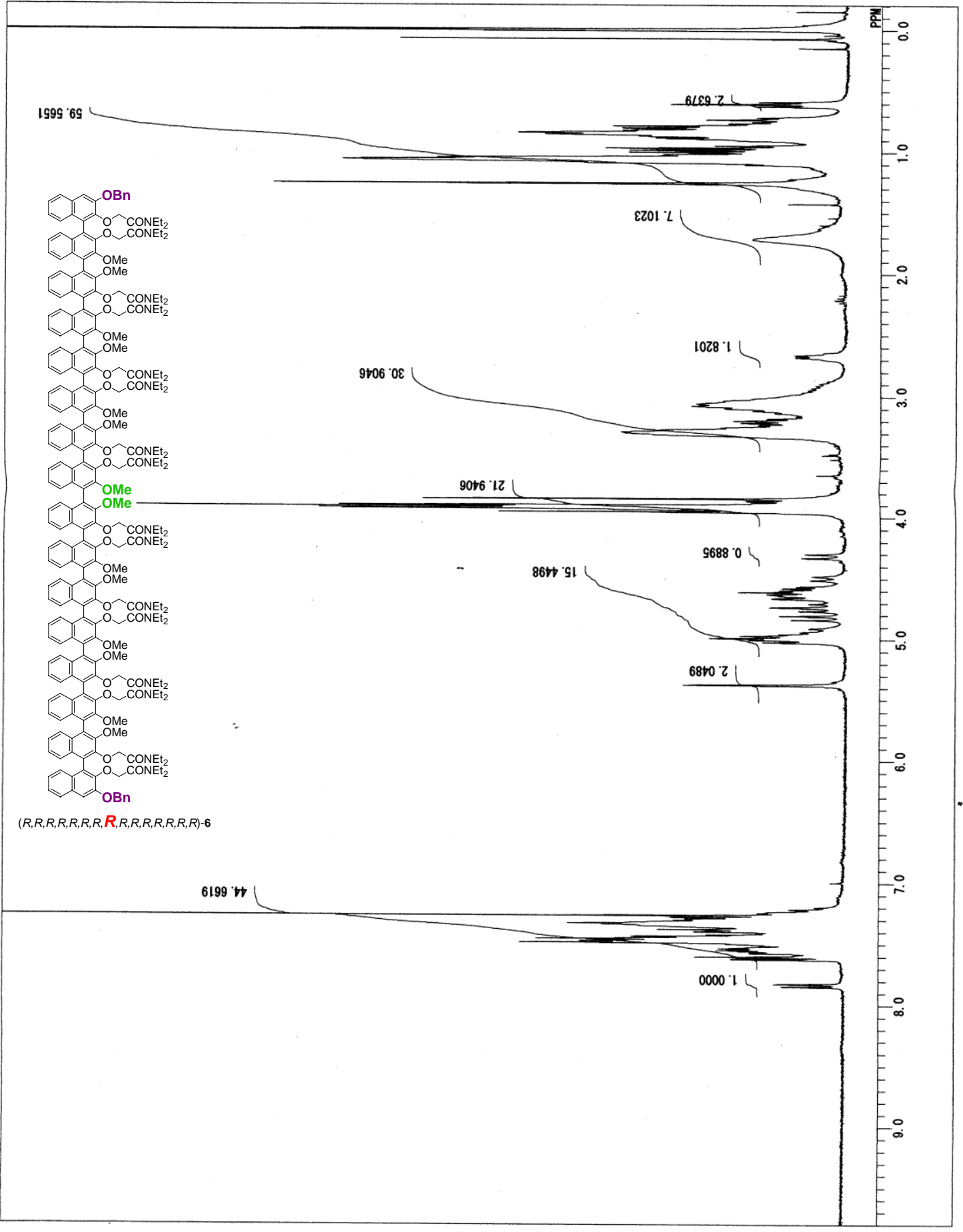




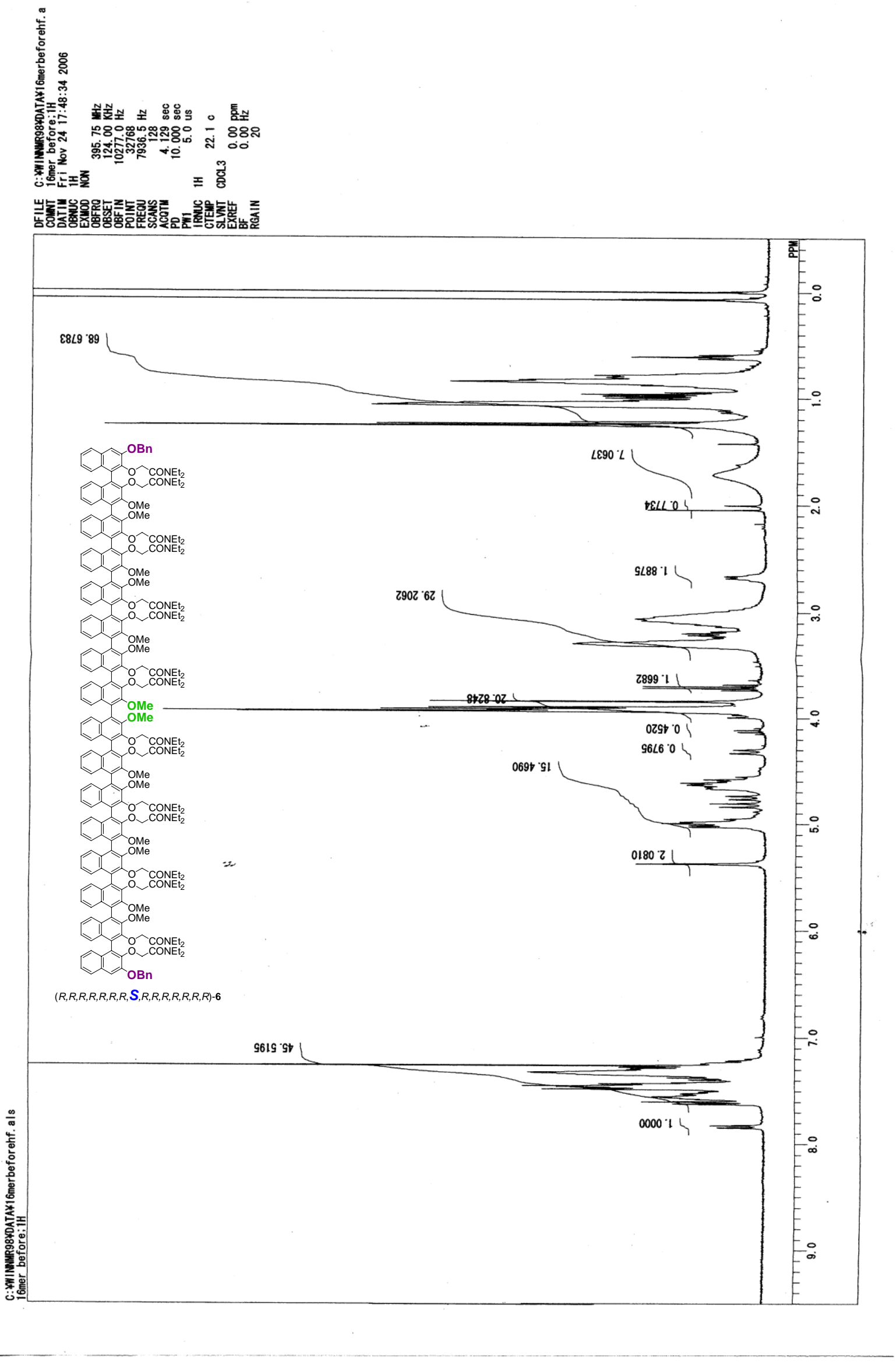




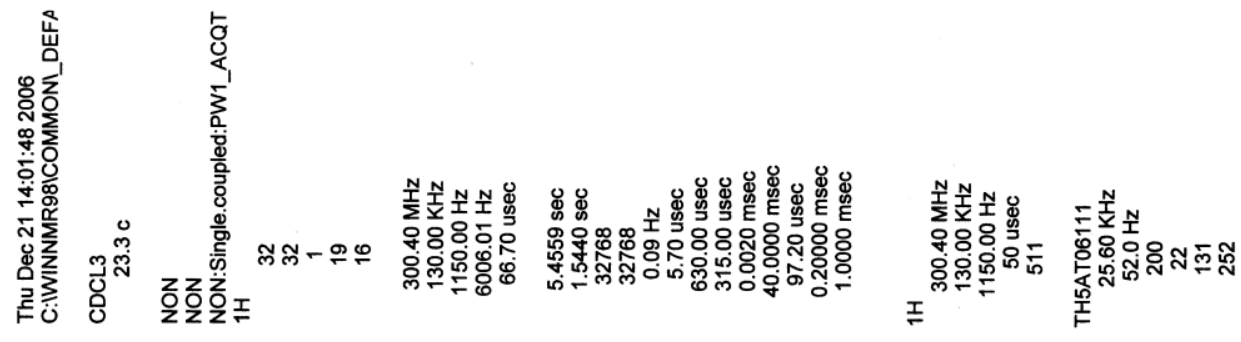

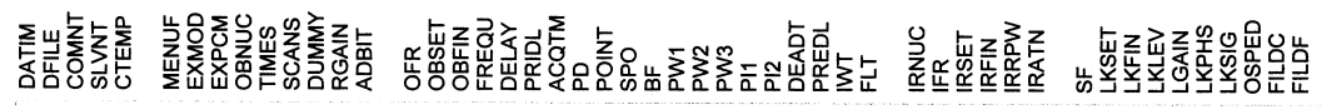

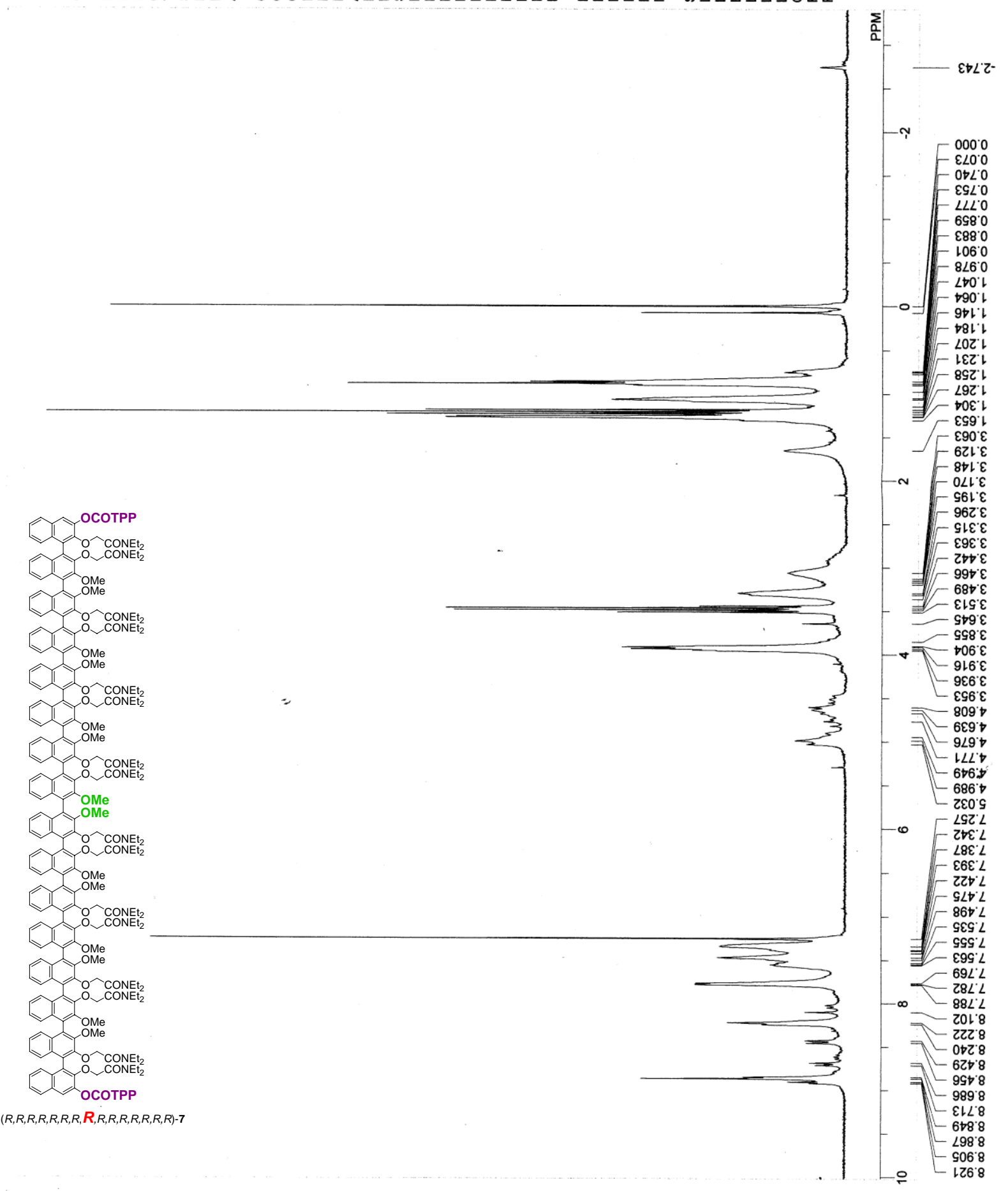




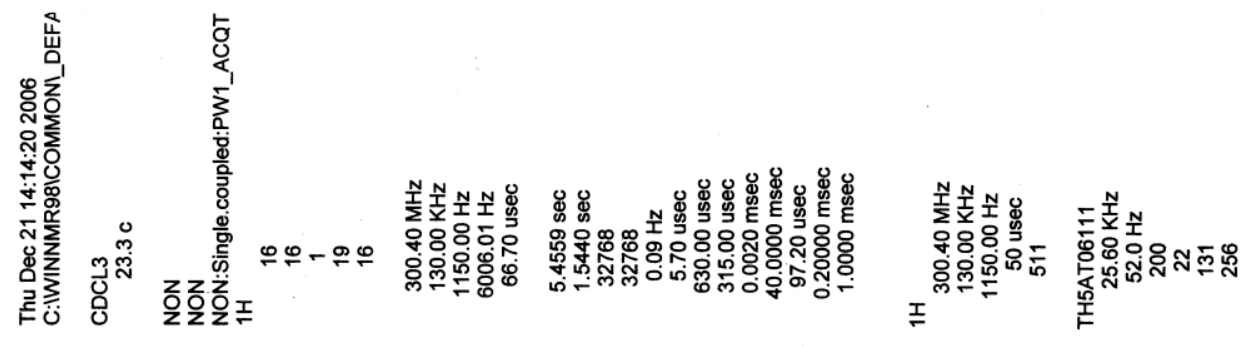

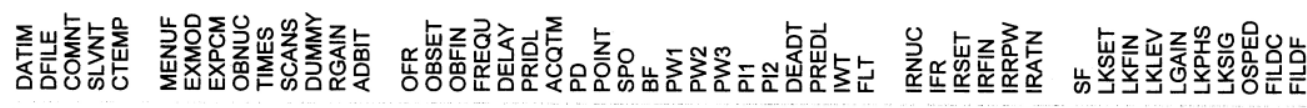

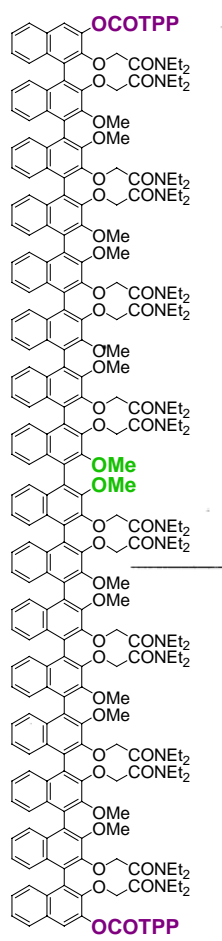



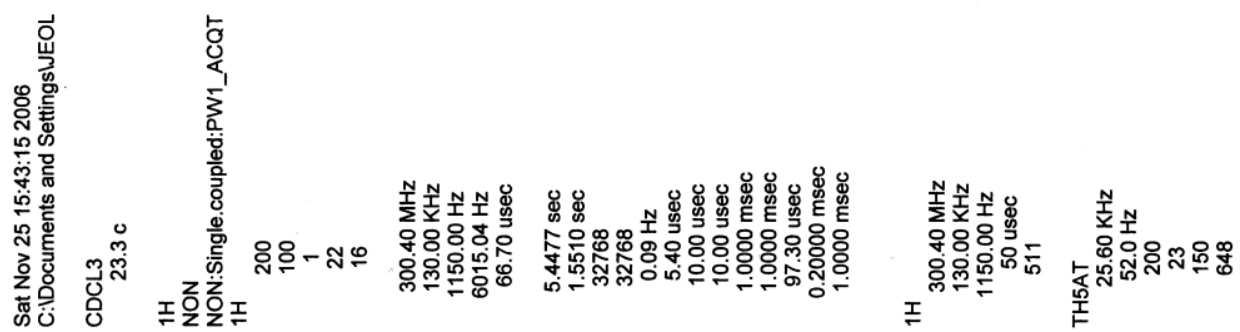

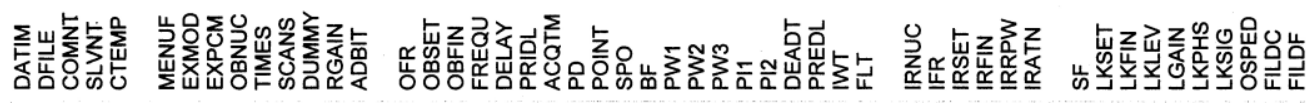

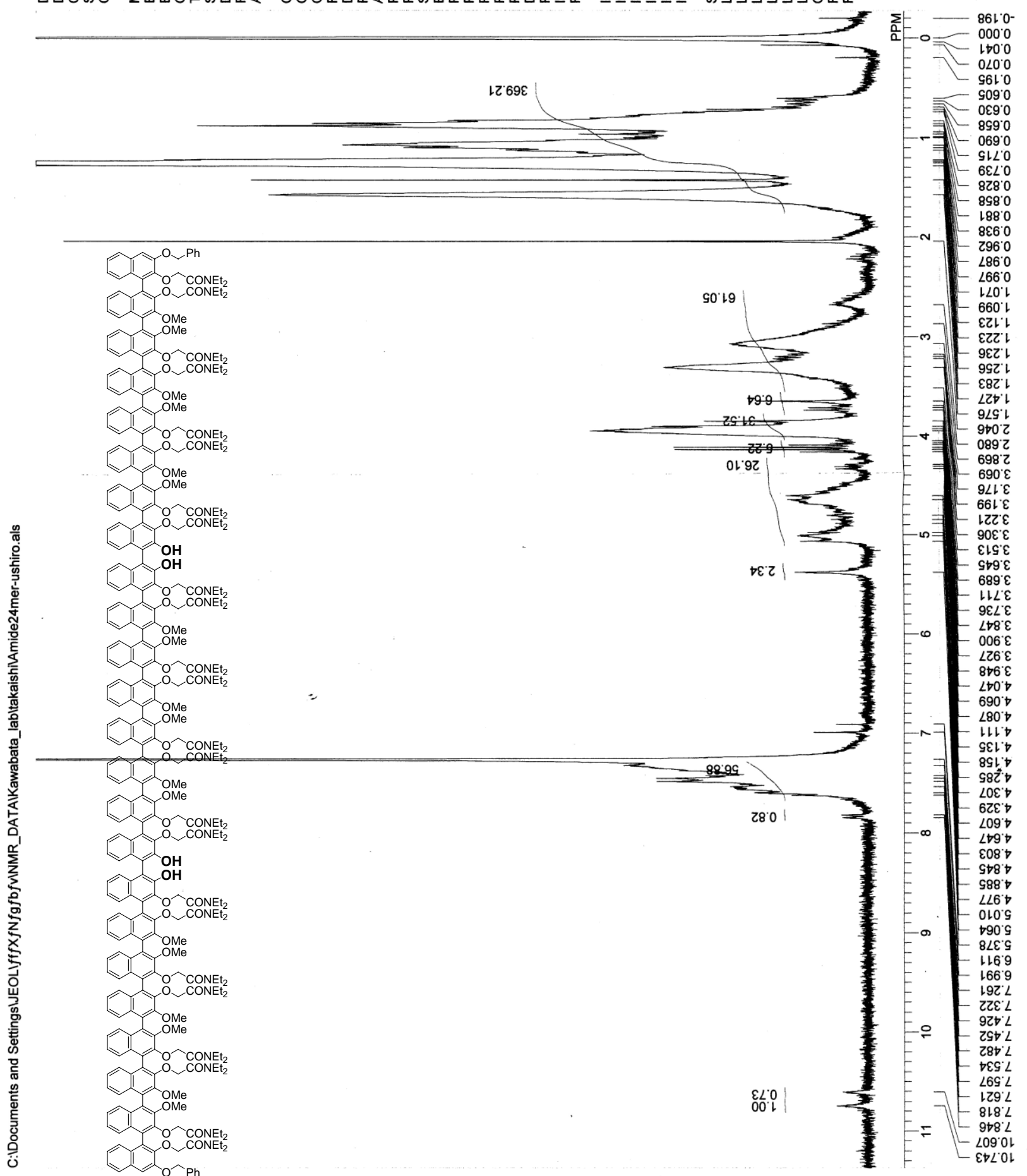




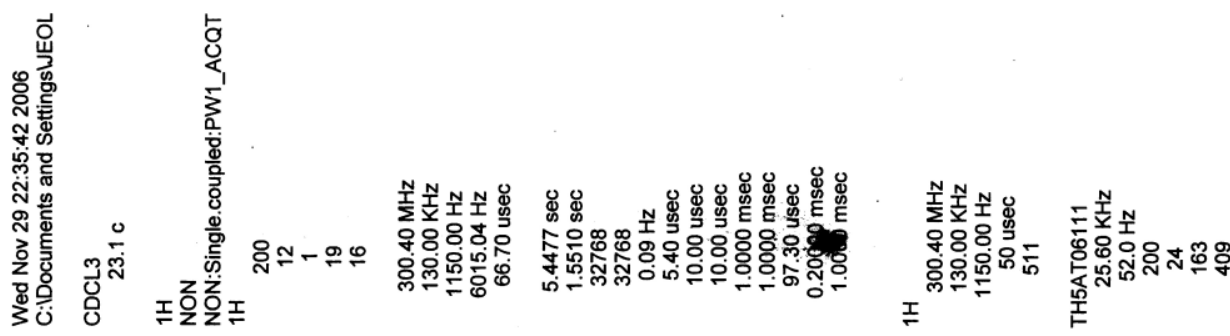

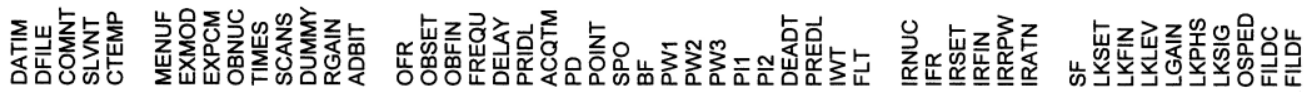

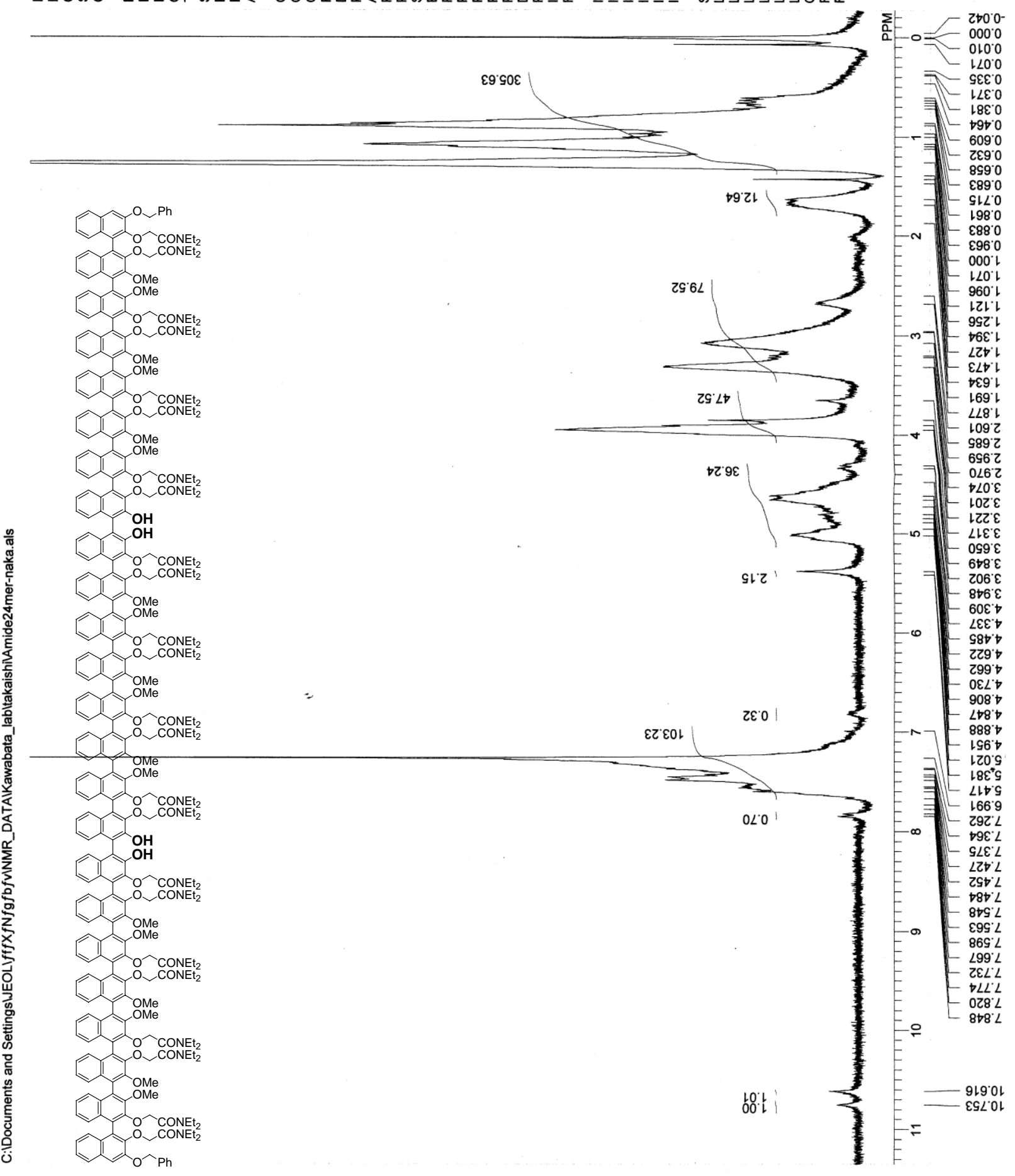

$(R, R, R, R, R, R, R, \boldsymbol{R}, R, R, R, R, R, R, R, \boldsymbol{S}, R, R, R, R, R, R, R)-\mathbf{9}$ 

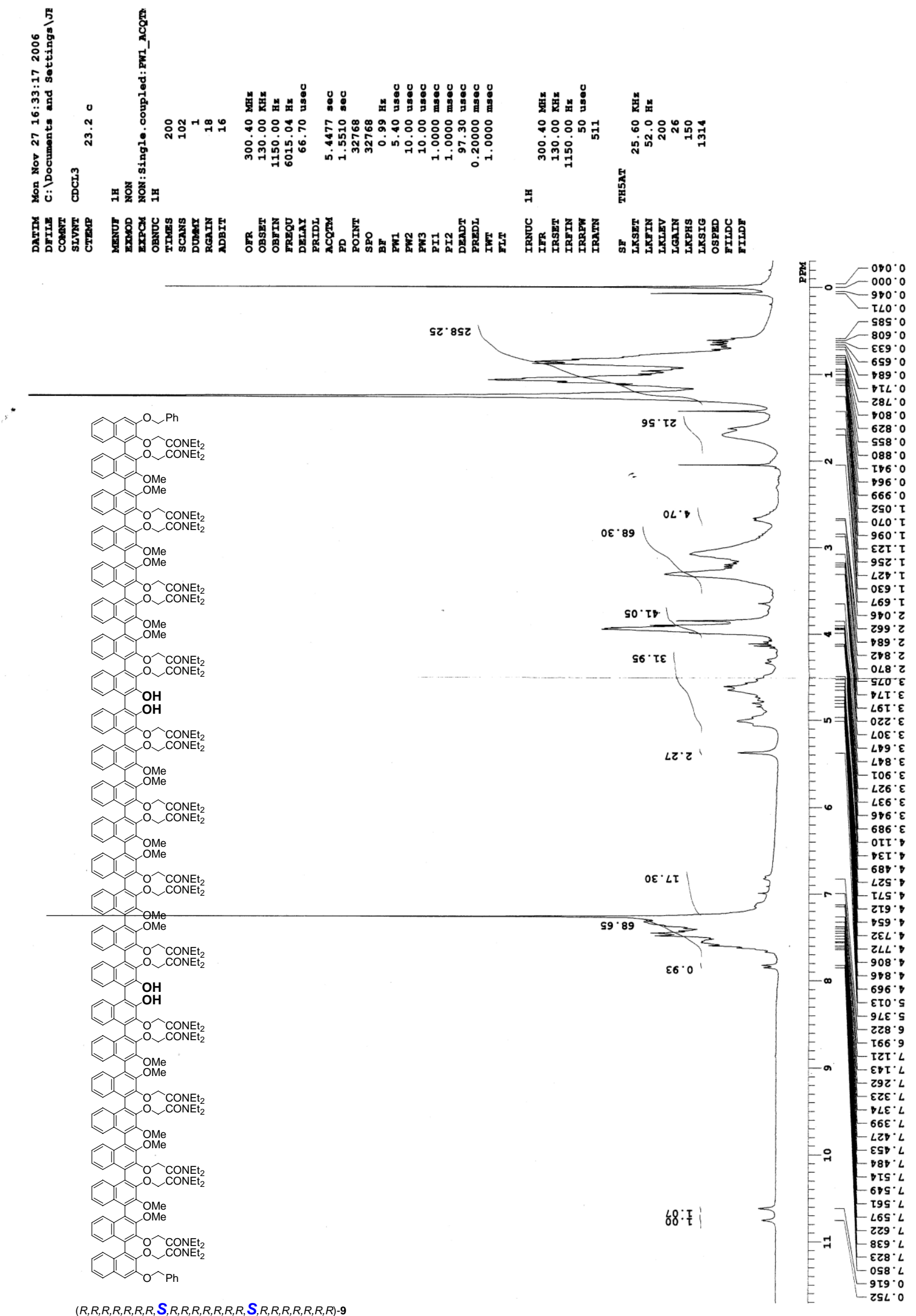


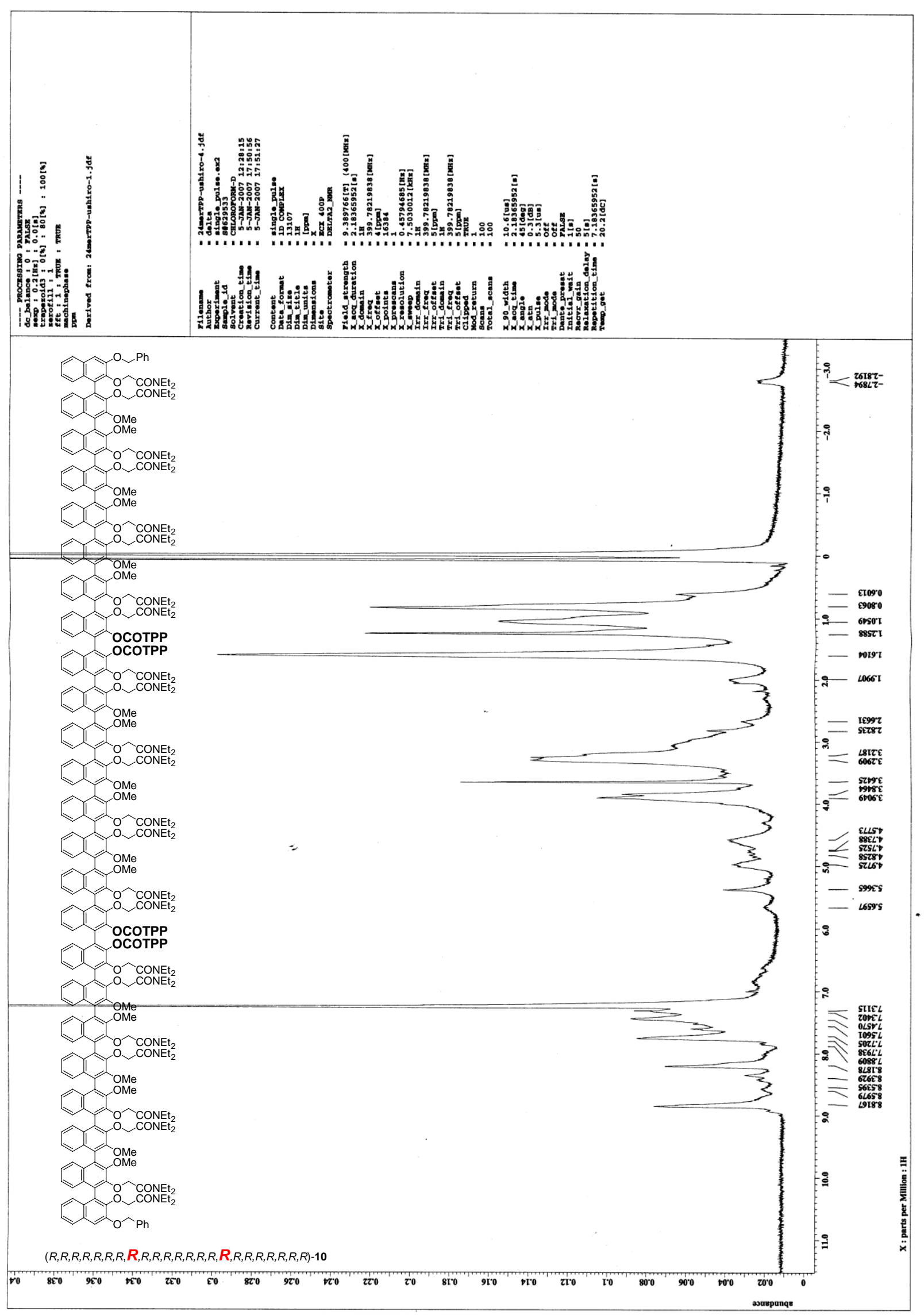




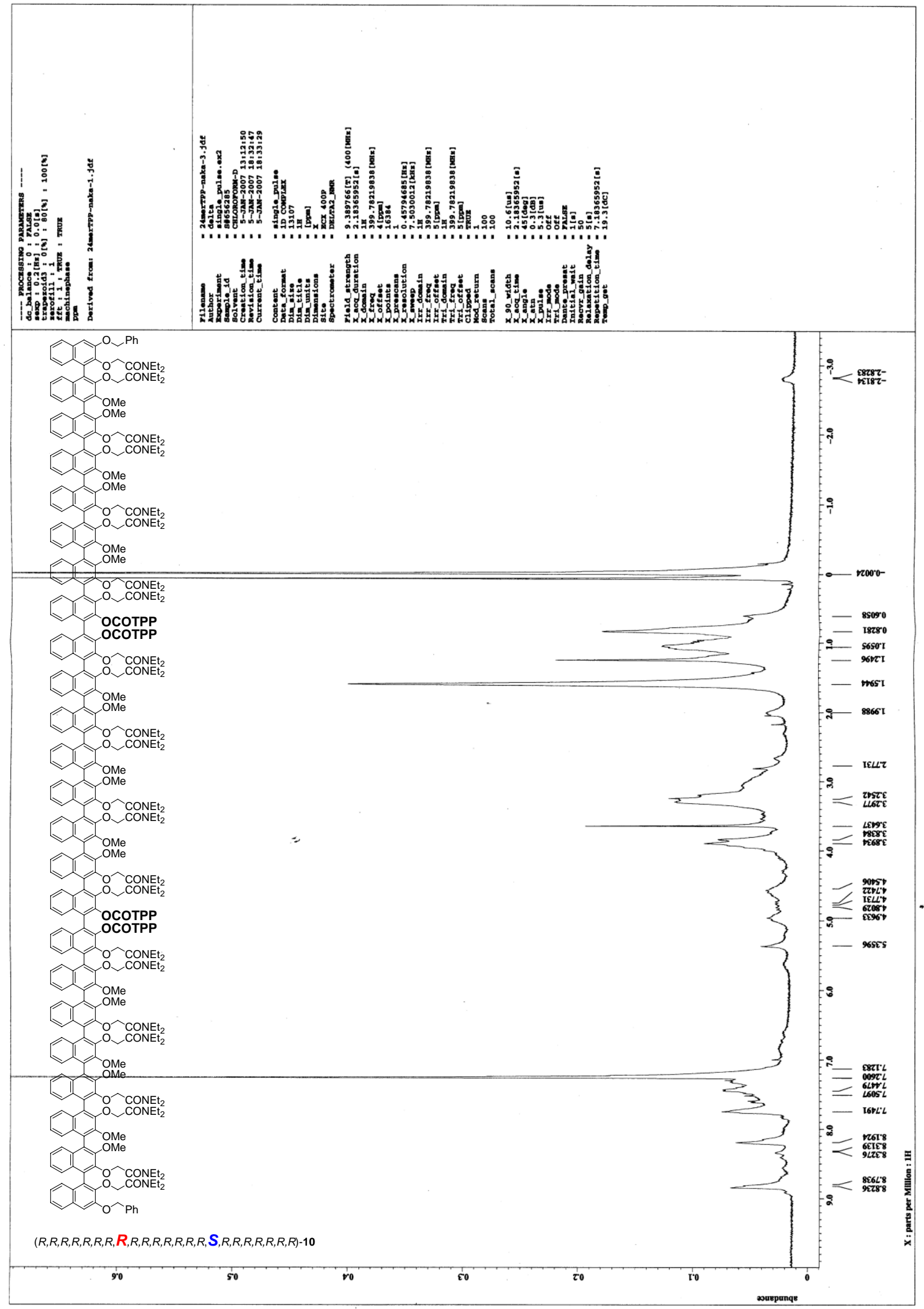




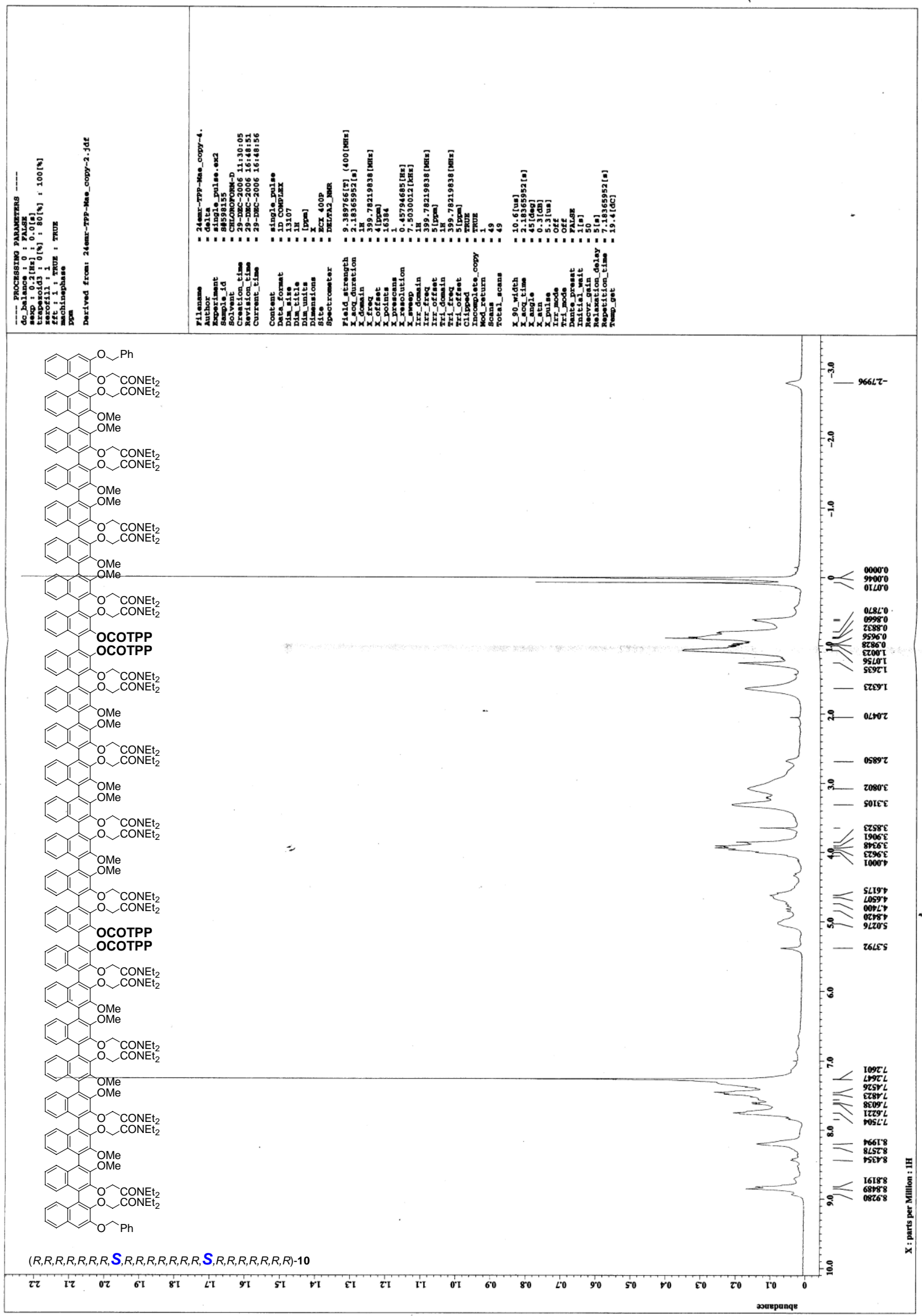




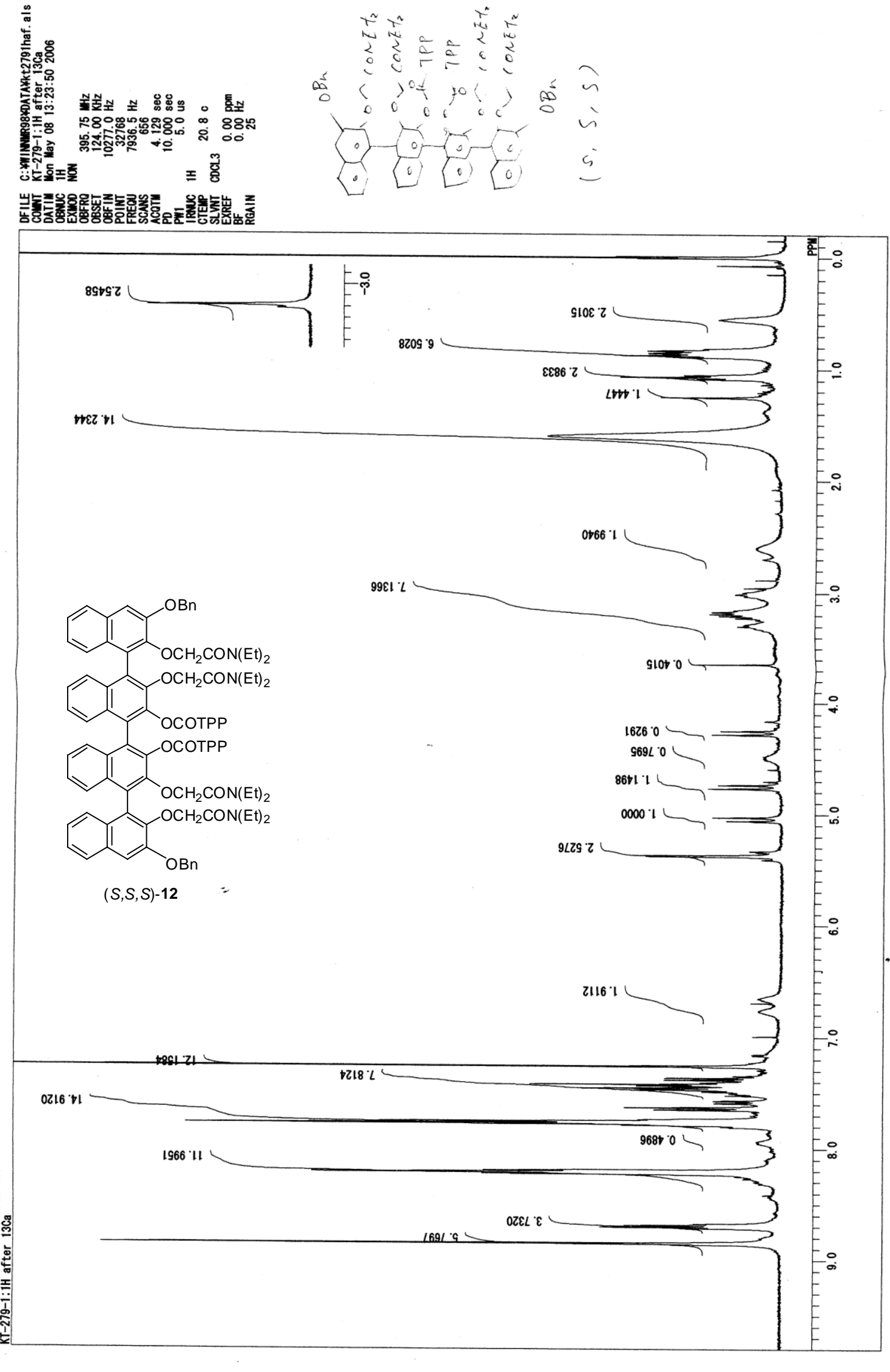




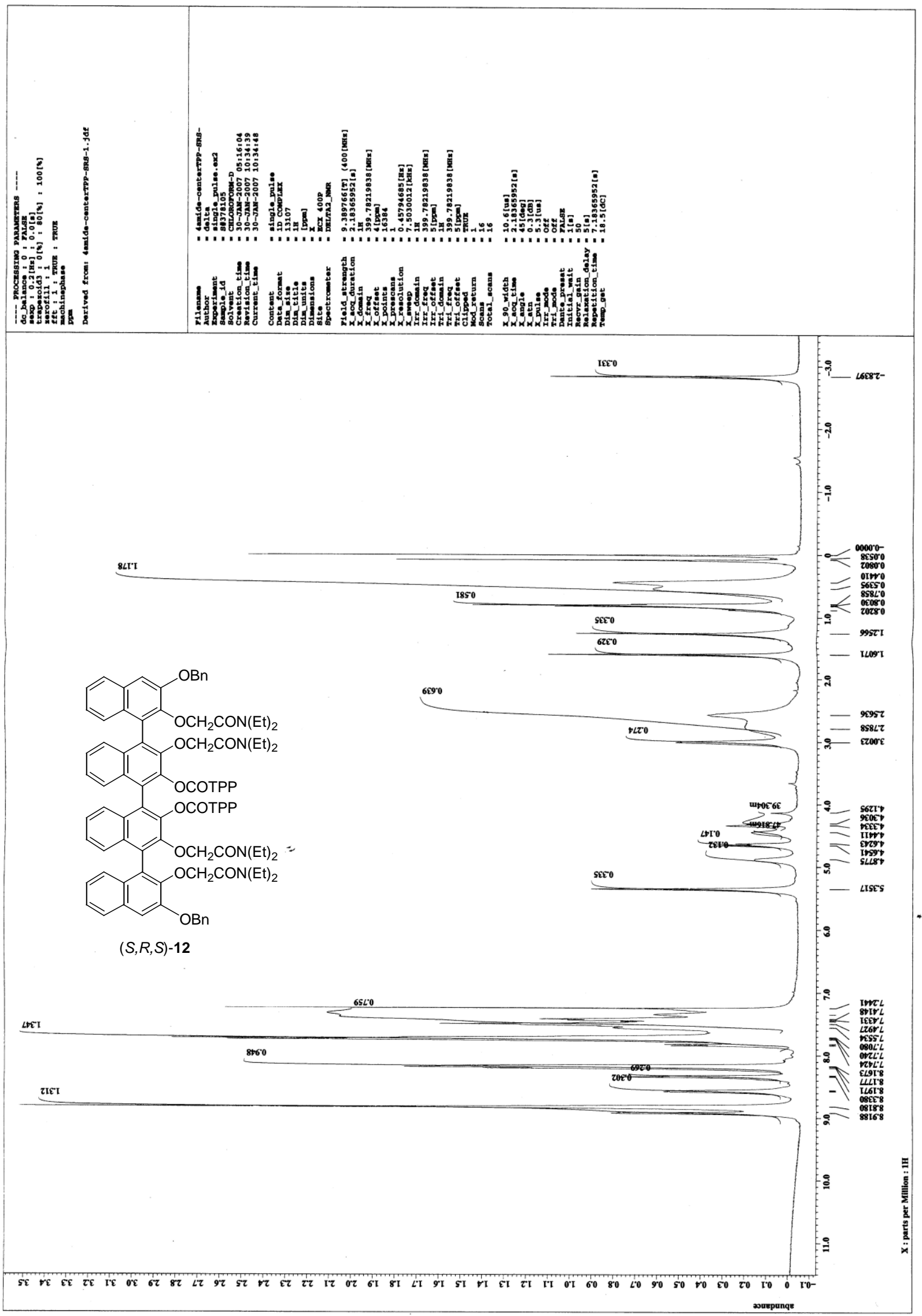


HPLC chart of the mixture of mono-ol 3 and diol 4.

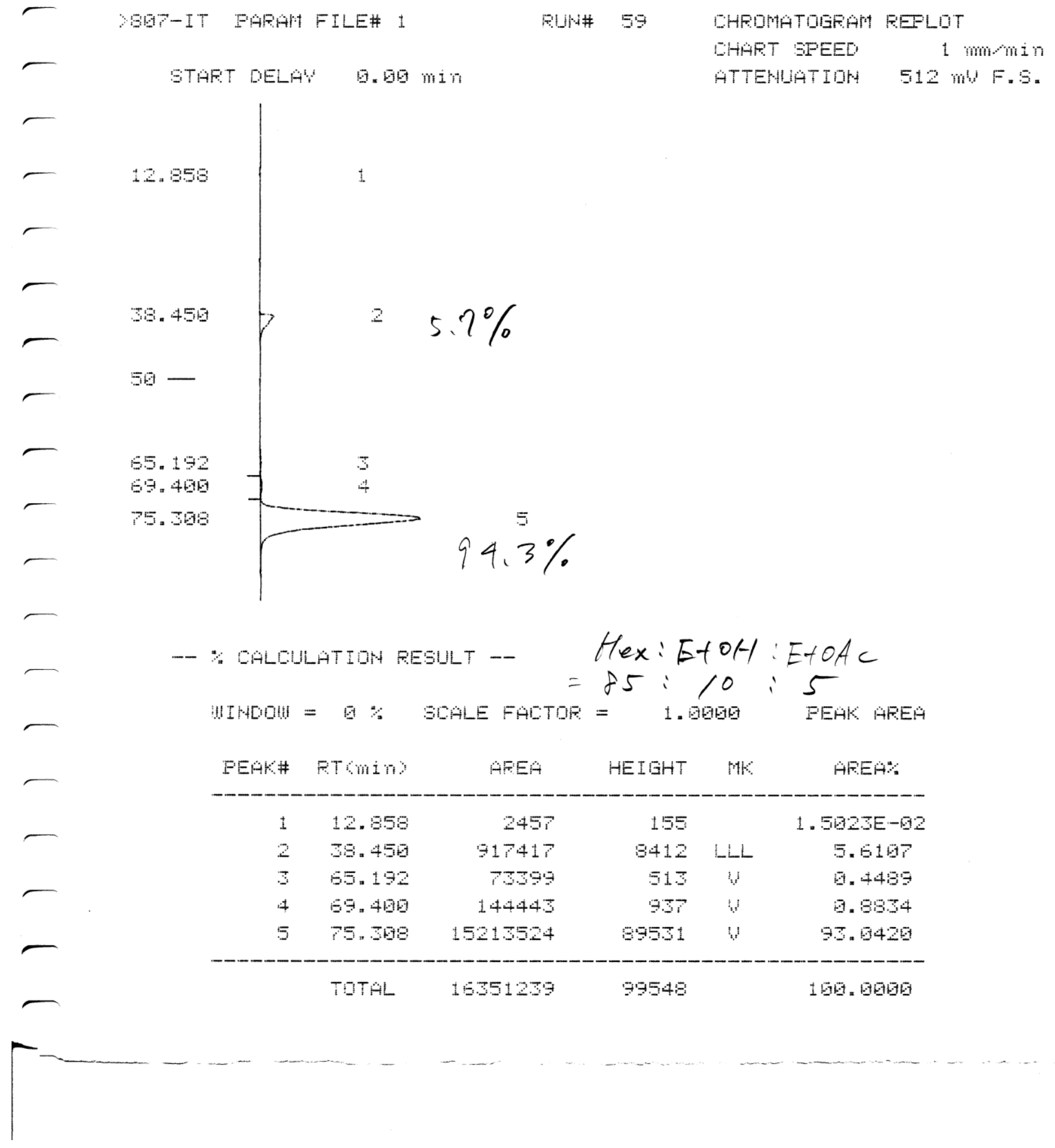

Conditions: COSMOSIL 5SL-II, 4.6 x $250 \mathrm{~mm}$ (nacalai tesque),

n-hexane:EtOH: EtOAc $=85: 10: 5, \quad 1.0 \mathrm{ml} / \mathrm{min}$ 